\title{
COMPARATIVE STUDY OF STEEL, NYLON 66 AND DELRIN HELICAL GEARS USED IN STEERING GEARBOX
}

\author{
Yogesh Ashok Ingale ${ }^{1}$, A. P. Kadam², D. G. Bhosale ${ }^{3}$ \\ ${ }^{1}$ M.E.[CAD/CAM/CAE] Student, Department of Mechanical Engineering, BVCOE Kolhapur, Maharashtra, India \\ ${ }^{2}$ Assistant Professor, Department of Mechanical Engineering, BVCOE Kolhapur, Maharashtra, India \\ ${ }^{3}$ Assistant Professor, Department of Automobile Engineering, Sanjeevan Engineering \& Tech. Institute Panhala, \\ Maharashtra, India
}

\begin{abstract}
This paper deals with designing of metallic \& nonmetallic material helical gear pair which is used for steering system. Plastics have found increasing use in automobile applications during the past 30 years. In this paper study review has be taken to understand the different properties of nonmetallic materials which are having greater advantages over metallic material.The nonmetallic material like Nylon 66 \& DELRIN acetal are taken as gear material. Normally steel (EN8) material gear pair is used in steering system. This paper is also deals with the percentage weight reduction in steering gear pair using materials like Nylon66 \& DELRIN.
\end{abstract}

Keywords: Steel, NYLON 66, DELRIN, AGMA tooth bending stress equation, etc.

\section{INTRODUCTION}

In today's era, the researchers from various automobile industries are doing wide research in composite and plastic material. So that, metal parts in vehicle can be replaced by composite material or by plastic material. In vehicle gears are used in the power transmission system and in the steering system. Gears are generally made up of steel, cast iron, etc. But recently composite and plastic materials were used,because they having sufficient mechanical properties like tensile strength, thermal strength, etc. These properties are similar to the steel, CI. Due to this reason metallic gear can be replaced by composite or plastic gear. Nylon 66 is the structure polymer material used for gears. But, polymer gears are used for low duty applications.

\section{PROBLEM DEFINITION}

The designing of different type of metallic and nonmetallic material gear pair to determine its strength under action of actual working conditions which are considered form specific automobile oriented case study.

\section{LITERATURE STUIDED}

Shekhar Pandurang Deokara, Ajay Kalmegh (2015) has mentioned that when metal gears are replaced by plastic gears in automobiles, appliances and machineries due to its advantages of low cost, lightweight, low noise, selflubrication, less wear, economic considerations, simple design and high production rate in manufacturing. Due to thermal wear nylon 6 and nylon 66 both gears failed, hence to overcome the problem of these gears the PA 46 is better option due to its superior properties and it also meet extreme performance challenges.
Laurentia Andrei, Gabriel Andrei, Alexandru Epureanu, Iulian Gabriel Birsan (2005) has concentrated on the plastic gears. They are mainly designed in such way that, which can replace the metallic gears by plastic gears, in order to enhance their transmissible power. Virtual models are used to investigate gear mesh, tooth deflections and strength, and a comparison to standard spur gears is drawn in their paper. Due to the complex gear geometry, a cutting process is appropriate for the gear manufacture.

\section{OBJECTIVE OF PROPOSED WORK}

This case study is to design nonmetallic material helical gear pair and to understand the different properties of nonmetallic materials which are having greater advantages over metallic material.

\section{MATERIAL PROPERTIES}

Before start to design any component one must know the different material properties which will define various design factors. The material properties of Nylon 66 material are as follows,

Table-1: Specifications of nylon 66 material

\begin{tabular}{|l|l|l|}
\hline $\begin{array}{l}\text { Sr. } \\
\text { No. }\end{array}$ & Parameter & $\begin{array}{l}\text { Nylon } \\
\mathbf{6 6}\end{array}$ \\
\hline 01 & Tensile Modules $\left(\mathrm{N} / \mathrm{mm}^{2}\right)$ & 2930 \\
\hline 02 & Tensile Strength $\left(\mathrm{N} / \mathrm{mm}^{2}\right)$ & 82.7 \\
\hline 03 & Flexural Yield Strength $\left(\mathrm{N} / \mathrm{mm}^{2}\right)$ & 103 \\
\hline 04 & Flexural Modulus $\left(\mathrm{N} / \mathrm{mm}^{2}\right)$ & 3102 \\
\hline 05 & Density $\left(\mathrm{kg} / \mathrm{m}^{3}\right)$ & 1150 \\
\hline 06 & Coefficient of Friction & 0.25 \\
\hline
\end{tabular}


The material properties of DELRIN material are as follows,

Table-2: Specifications of DELRIN material

\begin{tabular}{|l|l|l|}
\hline $\begin{array}{l}\text { Sr. } \\
\text { No. }\end{array}$ & Parameter & $\begin{array}{l}\text { DELRIN } \\
\text { acetal }\end{array}$ \\
\hline 01 & Tensile Modules $\left(\mathrm{N} / \mathrm{mm}^{2}\right)$ & 6200 \\
\hline 02 & Tensile Strength $\left(\mathrm{N} / \mathrm{mm}^{2}\right)$ & 101 \\
\hline 03 & Flexural Yield Strength $\left(\mathrm{N} / \mathrm{mm}^{2}\right)$ & 110 \\
\hline 04 & Flexural Modulus $\left(\mathrm{N} / \mathrm{mm}^{2}\right)$ & 6400 \\
\hline 05 & Density $\left(\mathrm{kg} / \mathrm{m}^{3}\right)$ & 1420 \\
\hline 06 & Coefficient of Friction & 0.2 \\
\hline
\end{tabular}

\section{DESIGN METHODLOGY}

The following steel, Nylon 66 \& DELRIN material gear pair was designed as per AGMA standard.

Table-3: Specifications of steel gear pair

\begin{tabular}{|l|l|l|l|}
\hline Sr. & Details Steel of Gear Pair \\
\cline { 2 - 4 } & Properties & Pinion & Gear \\
\hline 01 & Module $[\mathrm{m}]$ & $2.25 \mathrm{~mm}$ \\
\hline 02 & Pressure Angle $[\varnothing]$ & $20^{\circ}$ \\
\hline 03 & Helix Angle $[\varphi]$ & $30^{0}$ & \multicolumn{2}{|l|}{} \\
\hline 04 & No. of Teeth & 06 & 22 \\
\hline
\end{tabular}

\subsection{Bending Stress Equation for Steel (AGMA}

\section{Bending Stress)}

$$
\sigma=\mathrm{Wt} \times \mathrm{K}_{\mathrm{O}} \times \mathrm{Kv} \times \mathrm{Ks} \times \frac{\mathrm{K}_{\mathrm{m}} \times \mathrm{K}_{\mathrm{B}}}{\mathrm{J}} \times \frac{\mathrm{Pc}}{\mathrm{F}}
$$

Here,

1. Force acting tangentially on pinion $\left(\mathrm{W}^{\mathrm{T}}\right)=174.73 \mathrm{~N}$

2. Overload Factor Ko $=1.00$ for light shock load from standard table.

3. Dynamic Factor $K_{V}=\left\{\frac{A+\sqrt{200 V}}{A}\right\}^{B}(K v)=1.1$

4. Size Facto $\mathrm{K}_{\mathrm{S}}=0.904(\mathrm{~b} \times \mathrm{m} \times \sqrt{\mathrm{Y}})^{0.0535} \mathrm{~K}_{\mathrm{S}}=1.1$

5. Rim -Thickness Factor $\mathrm{K}_{\mathrm{B}}=1.6 \ln \left(2.242 / \mathrm{m}_{\mathrm{B}}\right)$

$$
\mathrm{K}_{\mathrm{B}}=1.00
$$

6. Load- Distribution Factor

$$
\mathrm{K}_{\mathrm{m}}=\left(\mathrm{C}_{\mathrm{mf}}\right)=1+\mathrm{C}_{\mathrm{mc}}\left(\mathrm{C}_{\mathrm{pf}} \mathrm{C}_{\mathrm{pm}}+\mathrm{C}_{\mathrm{ma}} \mathrm{C}_{\mathrm{e}}\right) \quad \mathrm{K}_{\mathrm{m}}=1.1
$$

Like this we get all the following standard factors.

Table-4: Specifications of the design gear pair

\begin{tabular}{|l|l|l|l|l|l|l|l|}
\hline Factor & $\mathbf{K}_{\mathbf{o}}$ & $\mathbf{K}_{\mathbf{v}}$ & $\mathbf{K}_{\mathbf{s}}$ & $\mathbf{J}$ & $\frac{\mathrm{Pc}}{\mathrm{F}}$ & $\mathbf{K}_{\mathbf{m}}$ & $\mathbf{K}_{\mathbf{B}}$ \\
\hline Value & 1.0 & 1.1 & 1.1 & 2.31 & 0.236 & 1.1 & 1.0 \\
\hline
\end{tabular}

$\sigma=174.73 \times 1.0 \times 1.1 \times 1.1 \times \frac{1.00 \times 1.00}{2.31} \times \frac{7.068}{30}$

$\sigma=21.61 \mathrm{MPa}$
Table-5: Specifications of nylon 66 gear pair

\begin{tabular}{|l|l|l|l|}
\hline \multirow{2}{*}{$\begin{array}{l}\text { Sr. } \\
\text { No. }\end{array}$} & Details Nylon 66 of Gear Pair \\
\cline { 2 - 4 } & Properties & Pinion & Gear \\
\hline 01 & Module $[\mathrm{m}]$ & $3.00 \mathrm{~mm}$ \\
\hline 02 & Pressure Angle $[\varnothing]$ & $20^{0}$ & \\
\hline 03 & Helix Angle $[\varphi]$ & $30^{0}$ & \\
\hline 04 & No. of Teeth & 07 & 21 \\
\hline
\end{tabular}

\subsection{Bending Stress Equation for Nylon 66 Material} (AGMA Bending Stress)

$$
\sigma=\mathrm{Wt} \times \mathrm{K}_{\mathrm{O}} \times \mathrm{Kv} \times \mathrm{Ks} \times \frac{\mathrm{K}_{\mathrm{m}} \times \mathrm{K}_{\mathrm{B}}}{\mathrm{J}} \times \frac{\mathrm{Pc}}{\mathrm{F}}
$$

Here,

1. Force acting tangentially on pinion $\left(\mathrm{W}^{\mathrm{T}}\right)=174.73 \mathrm{~N}$

2. Overload Factor $\mathrm{Ko}=1.50$ for light shock load from standard table.

3. Dynamic Factor $K_{V}=\left\{\frac{A+\sqrt{200 V}}{A}\right\}^{B}(K v)=1.3381$

4. Size Facto $\mathrm{K}_{\mathrm{S}}=0.904(\mathrm{~b} \times \mathrm{m} \times \sqrt{\mathrm{Y}})^{0.0535} \mathrm{~K}_{\mathrm{S}}=1.118$

5. Rim -Thickness Factor $\mathrm{K}_{\mathrm{B}}=1.6 \ln \left(2.242 / \mathrm{m}_{\mathrm{B}}\right)$

$$
\mathrm{K}_{\mathrm{B}}=1.00
$$

6. Load- Distribution Factor

$$
\mathrm{K}_{\mathrm{m}}=\left(\mathrm{C}_{\mathrm{mf}}\right)=1+\mathrm{C}_{\mathrm{mc}}\left(\mathrm{C}_{\mathrm{pf}} \mathrm{C}_{\mathrm{pm}}+\mathrm{C}_{\mathrm{ma}} \mathrm{C}_{\mathrm{e}}\right) \quad \mathrm{K}_{\mathrm{m}}=1.22
$$

Like this we get all the following standard factors.

Table-6: Specifications of the design gear pair

\begin{tabular}{|l|l|l|l|l|l|l|l|}
\hline Factor & $\mathbf{K}_{\mathbf{o}}$ & $\mathbf{K}_{\mathbf{v}}$ & $\mathbf{K}_{\mathbf{s}}$ & $\mathbf{J}$ & $\frac{\mathrm{Pc}}{\mathrm{F}}$ & $\mathbf{K}_{\mathbf{m}}$ & $\mathbf{K}_{\mathbf{B}}$ \\
\hline Value & 1.5 & 1.3381 & 1.118 & 2.28 & 0.224 & 1.22 & 1.00 \\
\hline
\end{tabular}

$\sigma=174.73 \times 1.5 \times 1.3380 \times 1.11866 \times \frac{1.2201 \times 1.2154}{2.2897} \times \frac{9.4241}{42}$

$\sigma=47.23 \mathrm{MPa}$

\section{Permissible AGMA Bending Stress}

$\sigma_{\text {all }}=\frac{S_{t}}{S_{F}} \frac{Y_{N}}{K_{T} K_{R}}$

$\sigma_{\text {all }}=(103 / 2) \times[1 /(1 \times 1)]$

$\sigma_{\mathrm{all}}=51.5 \mathrm{MPa}$

\section{Safety Factors (SF)}

$\mathrm{SF}=\frac{S_{t} Y_{N} / K_{T} K_{R}}{\sigma}, S_{t}=103 \mathrm{MPa}$

Tensile / Yield strength for Nylon 66 material.

$\mathrm{SF}=(103 * 1 / 1 * 1) / 47.23$ 
$\mathrm{SF}=2.18 \approx 2.2$

Then the design is safe.

Table-7: Specifications of delrin gear pair

\begin{tabular}{|l|l|l|l|}
\hline \multirow{2}{*}{ Sr. } & \multicolumn{4}{|l|}{ Details Nylon 66 of Gear Pair } & \multicolumn{2}{|l|}{} \\
\cline { 2 - 4 } & Properties & Pinion & Gear \\
\hline 01 & Module $[\mathrm{m}]$ & $2.5 \mathrm{~mm}$ & \\
\hline 02 & Pressure Angle $[\varnothing]$ & $20^{0}$ & \\
\hline 03 & Helix Angle $[\varphi]$ & $30^{0}$ & \multicolumn{2}{|l}{} \\
\hline 04 & No. of Teeth & 05 & 21 \\
\hline
\end{tabular}

\subsection{Bending Stress Equation for Delrin Material} (AGMA Bending Stress)

$$
\sigma=\mathrm{Wt} \times \mathrm{K}_{\mathrm{O}} \times \mathrm{Kv} \times \mathrm{Ks} \times \frac{\mathrm{K}_{\mathrm{m}} \times \mathrm{K}_{\mathrm{B}}}{\mathrm{J}} \times \frac{\mathrm{Pc}}{\mathrm{F}}
$$

Here,

1. Force acting tangentially on pinion $\left(\mathrm{W}^{\mathrm{T}}\right)=174.73 \mathrm{~N}$

2. Overload Factor Ko $=1.50$ for light shock load from standard table.

3. Dynamic Factor $\mathrm{K}_{\mathrm{V}}=\left\{\frac{\mathrm{A}+\sqrt{200 \mathrm{~V}}}{\mathrm{~A}}\right\}^{\mathrm{B}}(\mathrm{Kv})=1.1$

4. Size Facto $\mathrm{K}_{\mathrm{S}}=0.904(\mathrm{~b} \times \mathrm{m} \times \sqrt{\mathrm{Y}})^{0.0535} \mathrm{~K}_{\mathrm{S}}=1.11$

5. Rim -Thickness Factor $\mathrm{K}_{\mathrm{B}}=1.6 \ln \left(2.242 / \mathrm{m}_{\mathrm{B}}\right)$

$$
\mathrm{K}_{\mathrm{B}}=1.00
$$

6. Load- Distribution Factor

$$
\mathrm{K}_{\mathrm{m}}=\left(\mathrm{C}_{\mathrm{mf}}\right)=1+\mathrm{C}_{\mathrm{mc}}\left(\mathrm{C}_{\mathrm{pf}} \mathrm{C}_{\mathrm{pm}}+\mathrm{C}_{\mathrm{ma}} \mathrm{C}_{\mathrm{e}}\right) \quad \mathrm{K}_{\mathrm{m}}=1.00
$$

Like this we get all the following standard factors.

Table-8: Specifications of the design gear pair

\begin{tabular}{|l|l|l|l|l|l|l|l|}
\hline Factor & $\mathbf{K}_{\mathbf{o}}$ & $\mathbf{K}_{\mathbf{v}}$ & $\mathbf{K}_{\mathbf{s}}$ & $\mathbf{J}$ & $\frac{\mathrm{Pc}}{\mathrm{F}}$ & $\mathbf{K}_{\mathbf{m}}$ & $\mathbf{K}_{\mathbf{B}}$ \\
\hline Value & 1.5 & 1.1 & 1.1 & 2.30 & 0.262 & 1.00 & 1.00 \\
\hline
\end{tabular}

$\sigma=174.73 \times 1.5 \times 1.1 \times 1.1 \times \frac{1.00 \times 1.00}{2.30} \times \frac{7.86}{30}$

$\sigma=36.125 \mathrm{MPa}$

\section{Permissible AGMA Bending Stress}

$\sigma_{\text {all }}=\frac{s_{t}}{s_{F}} \frac{Y_{N}}{K_{T} K_{R}}$

$\sigma_{\text {all }}=(110 / 2) \times[1 /(1 \times 1)]$

$\sigma_{\text {all }}=55 \mathrm{MPa}$

\section{Safety Factors (SF)}

$$
\mathrm{SF}=\frac{S_{t} Y_{N} / K_{T} K_{R}}{\sigma}, S_{t}=103 \mathrm{MPa}
$$

Tensile / Yield strength for Nylon 66 material.

$\mathrm{SF}=(110 * 1 / 1 * 1) / 36.125$

$\mathrm{SF}=3.0$

Then the design is safe.

\section{RESULT AND DISCUSSION}

Table-9: Different materials results

\begin{tabular}{|l|l|l|l|}
\hline Parameter & Steel & Nylon 66 & DELRIN \\
\hline Bending Stress (N/mm $\mathbf{2})$ & 21.61 & 47.23 & 36.13 \\
\hline
\end{tabular}

From above calculations we came to conclude that, the following results are there.

1. The nylon 66 gear can bend at double loading condition than that of steel gear.

2. As per the design calculation the nylon 66 gear can take $51.5 \mathrm{MPa}$ load at same load that of steel.

Table-10: Weight Comparison in $\mathrm{KG}$

\begin{tabular}{|l|l|l|l|}
\hline Parameter & Steel & Nylon 66 & DELRIN \\
\hline Pinion & 0.124 & 0.032 & 0.05 \\
\hline Rack & 1.4 & 0.115 & 0.562 \\
\hline Total Weight & 1.524 & 0.147 & 0.612 \\
\hline
\end{tabular}

3. Percentage weight reduction of NYLON 66 helical pinion and rack is,

$$
\begin{aligned}
\% \text { weight reduction } & =\text { Wsteel }- \text { Wnylon66/Wsteel } \times 100 \\
& =1.524-0.147 / 1.524 \times 100 \\
& =90.35 \%
\end{aligned}
$$

Here it is observed that from calculations of $\%$ weight reduction, it is possible to achieve major objective of weight reduction by using Nylon 66 which 9 times light weight than steel. Total reduction of weight due to use of Nylon 66 is around $1.377 \mathrm{Kg}$.

4. Percentage weight reduction of DELRIN helical pinion and rack is,

$\%$ weight reduction $=$ Wsteel - Wnylon66 $/$ Wsteel $\times 100$

$$
\begin{aligned}
& =1.524-0.612 / 1.524 \times 100 \\
& =60.00 \%
\end{aligned}
$$

Here it is observed that from calculations of $\%$ weight reduction, it is possible to achieve major objective of weight reduction by using Nylon 66 which 9 times light weight than steel. Total reduction of weight due to use of Nylon 66 is around $0.9144 \mathrm{Kg}$.

\section{CONCLUSION}

8.1 This paper suggests that based on design analysis between steel, Nylon 66 and DELRIN one can use the nonmetallic material for automobile application. 
8.2 Byselecting appropriatenon-metallic material and by the designing of gear as per standard procedure the percentage weight reduction can also be achieved.

8.3 Since the bending stress level for Nylon 66, DELRIN is more than Effective load on gear tooth and also factor of safety for both bending stress is more than recommended 1.5. Hence, the design is safe.

8.4 It also come to know that the weight reduction will help to improve the performance of automotive application with less cost. (If, the gears are produced in larger in quantity). This case study will help to gives the idea of selection of appropriate plastic material, so that it can withstand the desired load and may use for various applications like steering system gear box.

\section{REFERENCES}

[1] Dr. Stefan Beermann "Estimation of lifetime for plastic gears" (2007)

[2] Shekhar Pandurang Deokara, Ajay Kalmegh " Design and Development of Polyamide 46 (PA46) Plastic gear in Engine Applications", International Journal Of Scientific Progress And Research (IJSPR) ISSN: 2349-4689, Volume-11, ( 2015)

[3] Laurentia Andrei, Gabriel Andrei, Alexandru Epureanu, Iulian Gabriel Birsan "Synthesis and Analysis of Plastic Curved Face width Spur Gears" VIII, ISSN 1221-4590 (2005)

[4] Mahebub Vohra, Prof. Kevin Vyas " Comparative Finite Element Analysis of Metallic and Non Metallic Spur Gear"(IOSR) Journal of Mechanical and Civil Engineering (IOSR-JMCE) e-ISSN: 2278-1684, pISSN: 2320-334X, Volume 11, Issue 3 Ver. IV , PP 136-145 (2014)

[5] Dr. Ir H.G.H. van Melick "Influence on load sharing stresses and wears, studied by FEA" (2007)

[6] T. Shoba Rani, T. Dada Khalandar "Spur gear" International Journal of Computational Engineering Research, Volume 03, Issue 11, ISSN: 22593005,(2013)

[7] A. D. Dighe, A. K. Mishra, V. D. Wakchaure "Investigation of Wear Resistance and Torque Transmission Capacity of Glass Filled Polyamide and PEEK Composite Spur Gears" International Journal of Engineering and Advanced Technology (IJEAT) ISSN: 2249 - 8958, Volume-3, Issue-3,(2014)

\section{BIOGRAPHIES}

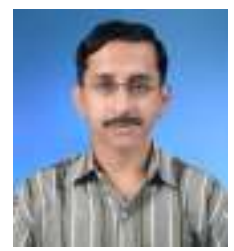

Mr. Yogesh Ashok Ingale, M.E. [CAD/CAM/CAE] Student, Department of Mechanical Engineering, BVCOE, Kolhapur.

ingaleyogesh20@gmail.com

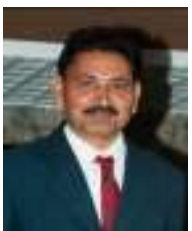

Prof. A. P. Kadam, Assistant Professor, Department of Mechanical Engineering, BVCOE, Kolhapur.

apkadam75@rediffmail.com

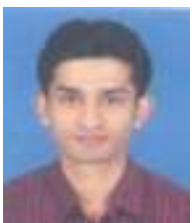

Prof. D. G. Bhosale, Assistant Professor, Department of Automobile Engineering, Sanjeevan Engineering \& Technology Institute, Panhala, Kolhapur. digvijay_bhonsale@yahoo.co.in 